\title{
Betriebliche Auswirkungen industrieller Servicerobotik am Beispiel der Kleinteilemontage
}

\author{
André Hengstebeck, Kirsten Weisner, Jochen Deuse, Jürgen Rossmann \\ und Bernd Kuhlenkötter
}

\subsection{Einleitung}

Kurze Produktlebenszyklen, volatile Kundenbedarfe und eine steigende Diversifizierung von Produkten und Prozessen führen in Kombination mit der steigenden Digitalisierung der Arbeitswelt im Kontext von Industrie $4.0 \mathrm{zu}$ erhöhten Flexibilitätsanforderungen in der Produktion. Das Ergebnis sind Arbeitsprozesse, die durch hohe Komplexität und eine gleichermaßen geringe Wiederholbarkeit geprägt sind. Aufgrund der adressierten Flexibilitätsanforderungen hat der Trend der Hochautomatisierung in den vergangenen Jahren stark nachgelassen (Beumelburg 2010). Stattdessen rücken im industriellen Umfeld insbesondere teilautomatisierte, hybride Arbeitssysteme in den Fokus. Unter Berücksichtigung bestehender Normen und Standards bzgl. Sicherheitsanforderungen und -konzepten (u. a. DIN EN ISO 10218 (Deutsches Institut für Normung e.V. 2012); DIN EN ISO 15536 (Deutsches Institut für Normung e.V. 2008); DIN EN ISO 20685 (Deutsches Institut

\footnotetext{
A. Hengstebeck $(\square) \cdot$ K. Weisner $\cdot$ J. Deuse

RIF e. V. Institut für Forschung und Transfer, Abteilung Produktionssysteme, Joseph-von-Fraunhofer-Str. 20, 44227 Dortmund, Deutschland e-mail: andre.hengstebeck@rif-ev.de; kirsten.weisner@rif-ev.de; jochen.deuse@ rif-ev.de; rossmann@ rif-ev.de; bernd.kuhlenkoetter@rif-ev.de

J.Rossmann

RIF e. V. Institut für Forschung und Transfer, Bereich Robotertechnik Joseph-von-Fraunhofer-Str. 20, 44227 Dortmund, Deutschland e-mail: rossmann@rif-ev.de

B.Kuhlenkötter

RIF e. V. Institut für Forschung und Transfer, Abteilung Produktionsautomatisierung Joseph-von-Fraunhofer-Str. 20, 44227 Dortmund, Deutschland e-mail: bernd.kuhlenkoetter@rif-ev.de
} 
für Normung e.V. 2010); ISO/TS 15066 (International Organization for Standardization 2016); BGIA 2011) gewinnt die direkte Mensch-Roboter-Kollaboration zunehmend an Bedeutung. Während anfänglich noch trennende Schutzeinrichtungen (z. B. Schutzzaun) eine konsequente Teilung der Arbeitsräume des Menschen und des Roboters zur Folge hatten, wird derzeit eine direkte, kontinuierliche Zusammenarbeit im Rahmen eines gemeinsamen Arbeitsbereichs angestrebt (Spingler und Thiemermann 2001).

Ein in diesem Kontext zentrales Konzept ist aktuell die industrielle Servicerobotik. Die Servicerobotik im Allgemeinen ist in verschiedenen Branchen und Anwendungsfeldern bereits ein fester Bestandteil (z. B. Sanierung von Abwasserrohren, Übernahme von Holund Bringdiensten in der Kranken- und Altenpflege) (Klöckner et al. 2015). Nach einer Definition des Bundesministeriums für Wirtschaft und Energie (BMWi) umfasst sie die Erbringung einer Dienstleistung für den Menschen durch den Roboter. Dabei agiert letzterer entweder in direkter Kollaboration mit dem Menschen oder völlig autonom. Ein besonderes Kennzeichen servicerobotischer Lösungen ist deren Mobilität und flexible Verwendbarkeit (Bundesministerium für Wirtschaft und Technologie 2013; Klöckner et al. 2015). In Anlehnung an die vorherige Definition beschreibt die industrielle Servicerobotik die Unterstützung des Menschen bei seiner Tätigkeit im industriellen Umfeld. Die arbeitsteilige Aufgabendurchführung ermöglicht hierbei eine Steigerung der Produktqualität und Prozessstabilität. Ferner besteht die Möglichkeit, Mitarbeiter von monotonen und körperlich belastenden Aufgaben (manuelle Lastenhandhabung, Einnahme von Gelenkgrenzstellungen u. a.) zu befreien und so $\mathrm{zu}$ einer gesundheitsförderlichen Arbeitsgestaltung beizutragen (Deuse et al. 2014b). Zusammengefasst generiert der mobile Einsatz servicerobotischer Lösungen somit eine erhöhte Flexibilität und Autonomie (Klöckner et al. 2015).

\subsection{Industrielle Servicerobotik in der Kleinteilemontage}

Zur ganzheitlichen Erschließung dieser Potenziale existieren bereits erste technische Gestaltungslösungen. In diesem Zusammenhang scheinen z. B. sensitive Leichtbauroboter, die auf Grundlage von Kraft-Momenten-Sensoren geregelt werden, für den Einsatz als industrielle Serviceroboter geeignet zu sein (Steegmüller und Zürn 2014; Universal Robots 2014). Trotz der Verfügbarkeit servicerobotischer Systeme ist der Umsetzungsgrad entsprechender Lösungen in realen Anwendungsszenarien allerdings bisher noch vergleichsweise gering. Eine mögliche Ursache besteht dabei in der Unsicherheit darüber, für welche Anwendungsszenarien und Prozessschritte sich derartige Robotersysteme konkret eignen und welche fallspezifischen Vorteile sich hieraus ergeben können. Zur Erleichterung der Entscheidungsfindung können Anwender auf eine Vielzahl unterschiedlicher Verfahren zur Analyse und Bewertung der Automatisierungseignung zurückgreifen, welche die zuvor formulierten Fragestellungen jedoch nur bedingt auflösen können (z. B. Beumelburg 2010; Parasuraman et al. 2000; Ross 2002; Schraft et al. 1993).

Auf dieser Grundlage besteht das Ziel des vom BMWi geförderten Forschungsprojekts MANUSERV in der Entwicklung einer webbasierten Planungsumgebung, welche die Potenziale industrieller Robotersysteme mit den spezifischen Anforderungen manueller 
Arbeitssysteme und -prozesse verknüpft. Auf diese Weise sollen dem industriellen Anwender konkret implementierbare, (teil-)automatisierte Gestaltungslösungen sowie die entsprechenden Ablauffolgen vorgeschlagen werden. Unter der Prämisse ein möglichst ganzheitliches Assistenzsystem bereitzustellen, besteht darüber hinaus die Möglichkeit, dem Anwender entscheidungsrelevante Zielgrößeninformationen bereitzustellen (z. B. Preis) (Deuse et al. 2014a). Zur Sicherstellung einer möglichst breiten industriellen Anwendbarkeit werden das Konzept und dessen softwaretechnische Implementierung anhand von drei realen, manuellen Arbeitsprozessen aus unterschiedlichen Anwendungsgebieten validiert. Dabei handelt es sich um einen Reinigungsprozess aus dem Bereich der Landwirtschaft, einen Umrüstvorgang aus dem Maschinen- und Anlagenbau sowie einen manuellen Montageprozess im Kontext der Elektro-Installationstechnik. Angelehnt an die im Rahmen von MANUSERV verfolgte Zielstellung wird der adressierte Montageprozess detailliert vorgestellt, um im darauffolgenden Schritt spezifische Automatisierungspotenziale abzuleiten.

Bei dem Anwendungsfall handelt es sich um die Montage eines Einbauradios mit Touch-Display, welches auf Basis einer konkreten Produktspezifikation des Kunden hergestellt wird. Aktuell erfolgt die Montage rein manuell an zwei nebeneinander angeordneten Arbeitsplätzen durch einen einzelnen Mitarbeiter. Die Montage des Radios kann grundsätzlich in drei übergeordnete Prozessschritte unterteilt werden. Hierzu gehören die Montage des Netzteils, die Montage des Bedienmoduls sowie die Montage des Lautsprechers. Daran anschließend erfolgt die Verpackung des Einbauradios.

Wie in Abb. 4.1 dargestellt, findet die Montage des Bedienmoduls am ersten Arbeitsplatz statt. Dies beinhaltet neben dem reinen Zusammenbau der Komponenten auch das Aufspielen der aktuellen Software sowie die abschließende Prüfung aller Funktionen durch ein spezielles Prüfgerät. Der Arbeitsinhalt am zweiten Arbeitsplatz besteht aus der Montage des Netzteils und des Lautsprechermoduls. Ein wesentlicher Prozessschritt ist in diesem Fall die erforderliche Laserbeschriftung des Netzteils, welche sowohl von der Vorder- als auch von der Rückseite zu erfolgen hat. Der Prozess endet mit der Verpackung
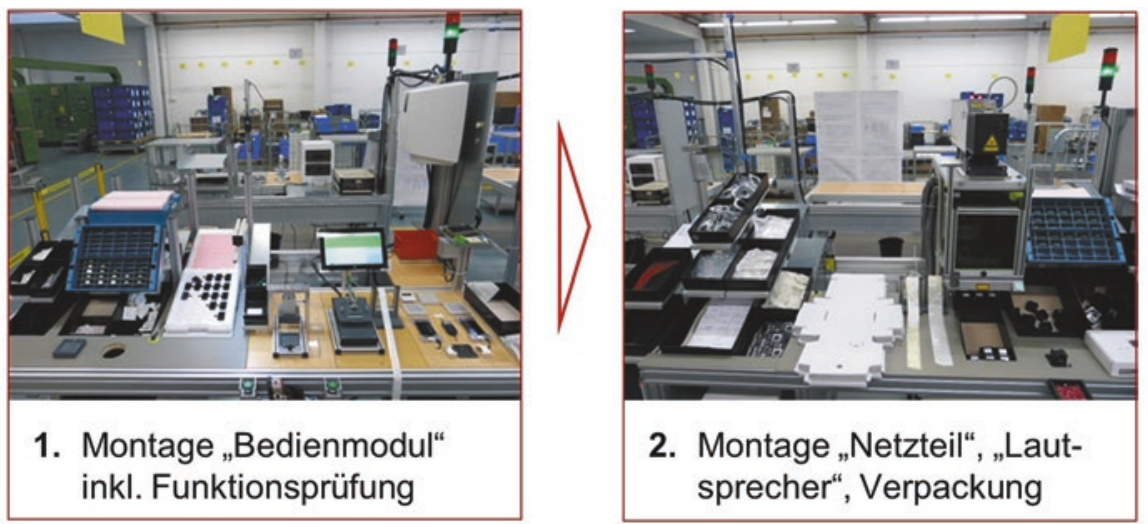

Abb. 4.1 Montage des Einbauradios 
des Einbauradios inkl. Bedienungsanleitung in einem Faltkarton. Die Verpackung selbst umfasst die Vorbereitung bzw. das Auffalten des Kartons, das Einlegen der einzelnen Komponenten (Bedienmodul, Netzteil, Lautsprechermodul) sowie das abschließende Aufkleben von Etiketten (Qualitätssiegel, Produktinformationen).

\subsection{Entwicklung eines hybriden Gestaltungskonzepts}

Am Beispiel des vorgestellten Montage-Szenarios wird im Folgenden eine Herangehensweise zur Entwicklung hybrider Gestaltungskonzepte betrachtet, welche sich an einer REFA-Methode der Arbeitssystemgestaltung orientiert und in Abb. 4.2 dargestellt ist (REFA Bundesverband e.V. 2013). Ausgehend von einem bestehenden manuellen Arbeitsplatz erfolgt hierbei zunächst eine umfassende Datenaufnahme. Anschließend werden wesentliche Anforderungen analysiert, welche eine Grundlage für die Grob- und Feinplanung des hybriden Konzepts bilden.

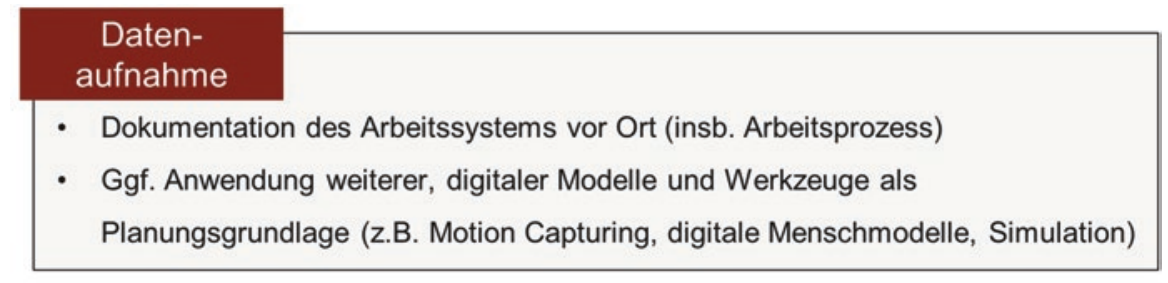

\section{Anforderungs-}

analyse

- Analyse von Verbesserungsmöglichkeiten durch industrielle Servicerobotik (z.B. Bewegungsökonomie, Monotonieabbau)

- Analyse erforderlicher Rahmenbedingungen zur Integration teilautomatisierter Systeme (z.B. Handhabung, Infrastruktur)

\section{Planung}

- Zusammenfassende Betrachtung der erkannten Verbesserungsmöglichkeiten und Rahmenbedingungen

- Erarbeitung eines hybriden Gestaltungskonzeptes

Abb. 4.2 Vorgehensweise zur Entwicklung einer Gestaltungsalternative 
Im ersten Schritt der Datenaufnahme werden sowohl der bestehende Ist-Arbeitsplatz als auch der entsprechende Arbeitsprozess vor Ort umfassend dokumentiert. Neben einer analogen Datenaufnahme (z. B. anhand des REFA-Arbeitssystems) können hierfür auch leistungsfähige digitale Werkzeuge zum Einsatz kommen. Für den skizzierten Anwendungsfall konnte z. B. die Anwendung von Motion Capturing dazu beitragen, menschliche Bewegungsdaten zu generieren und auf ein digitales Menschmodell zu übertragen. Dieses wurde unter Berücksichtigung aller Arbeitsplatzkomponenten in ein Simulationsmodell überführt, welches insgesamt eine zentrale Planungsbasis darstellt (s. Abb. 4.3).

Ausgehend von den aufgenommenen Ist-Daten kann die anschließende Anforderungsanalyse insbesondere dazu beitragen, Verbesserungsmöglichkeiten wie auch erforderliche Rahmenbedingungen für den Einsatz industrieller Servicerobotik im Kontext eines hybriden Gestaltungskonzeptes zu identifizieren. Konkrete Optimierungspotenziale ergeben sich für den vorgestellten Anwendungsfall u. a. dadurch, dass ein Mitarbeiter für die Durchführung der kompletten Arbeitsaufgabe verantwortlich ist. Die hierdurch entstehenden Laufwege des Mitarbeiters entsprechen aus bewegungsökonomischer Sicht vermeidbaren, nicht wertschöpfenden Tätigkeiten (Ohno 2009). Darüber hinaus ist der manuelle Arbeitsablauf durch viele Wiederholungen gekennzeichnet, was sich sowohl physiologisch als auch psychologisch negativ auf den Mitarbeiter auswirken kann (Schlick et al. 2010). Als Rahmenbedingungen zur Implementierung einer hybriden Gestaltungslösung konnten speziell die Bereitstellung der Arbeitsobjekte (z. B. vermischt liegend, vereinzelt) sowie die Form und Handhabbarkeit der Bauteile identifiziert werden. Zudem stellt die Verfügbarkeit von Stellflächen und der technischen Infrastruktur eine wichtige Voraussetzung für die Implementierung eines Robotersystems dar.

Bei der Grob- und Feinplanung wird unter Berücksichtigung aller aufgestellten Anforderungen und Rahmenbedingungen ein integriertes, hybrides Gestaltungskonzept entwickelt. Dieser Vorgehensweise entsprechend wurde die Entscheidung getroffen, zunächst

Abb. 4.3 Simulation auf Basis von Motion Capturing

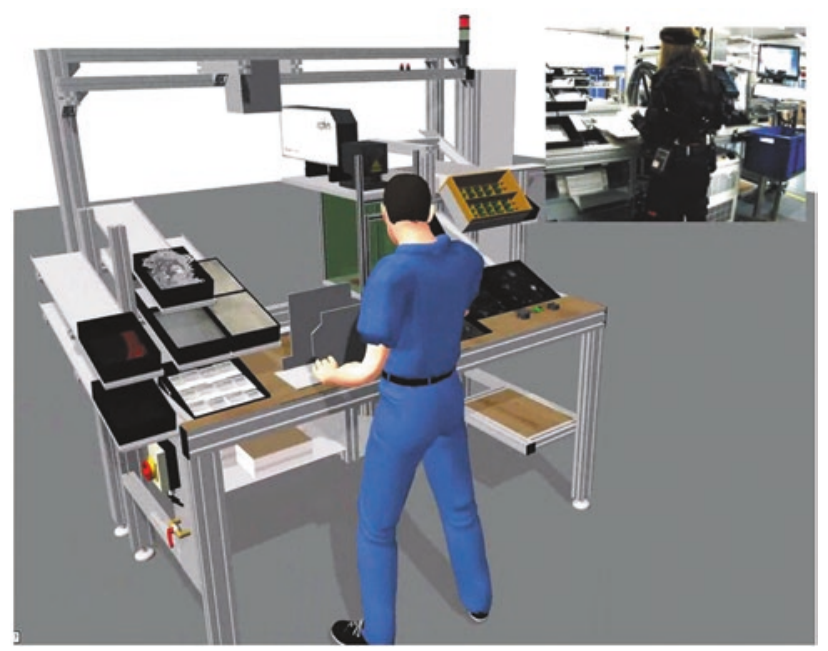


die Beschriftung des Netzteils mit Unterstützung eines Leichtbauroboters durchzuführen. Im Zuge einer anschließenden, ersten Integration und Nutzung eines Robotersystems im Produktionsbetrieb sind zahlreiche Auswirkungen auf die involvierten Mitarbeiter möglich, welche im Folgenden detailliert diskutiert werden.

\subsection{Mögliche Auswirkungen des hybriden Systems auf betriebliche Rollen am Beispiel der Kleinteilemontage}

Die technische Implementierung der vorgestellten Gestaltungsalternative kann sowohl für direkt als auch indirekt ausgerichtete betriebliche Rollen mit deutlichen Auswirkungen und Veränderungen verbunden sein. Im produktiven Bereich des Unternehmens sind dabei insbesondere die Rollen des Monteurs, des Meisters und des Instandhalters betroffen. Im indirekten Bereich des Unternehmens können spürbare Änderungen vor allem für Mitarbeiter auftreten, die in Verbindung mit der Montage stehen, wie z. B. Prozessplaner und Konstrukteure. Im Folgenden wird anhand verschiedener Bewertungskriterien untersucht, ob und inwiefern sich für die vorgestellten Bereiche Auswirkungen ergeben können (s. Abb. 4.4). Hierfür wurden die in diesem Buch vorgestellten Bewertungskriterien herangezogen und anwendungsfallspezifisch angepasst.

Für die Rolle des Monteurs kann die Integration industrieller Leichtbauroboter zahlreiche produktionsbetriebliche und mitarbeiterseitige Auswirkungen haben. So können autonome, industrielle Serviceroboter dazu genutzt werden, wiederholt und kontinuierlich auszuführende Prozessschritte an einem der beiden Arbeitsplätze zu übernehmen, um so die Anzahl nicht wertschöpfender Laufwege durch den Mitarbeiter zu reduzieren. Auf diese Weise kann der Arbeitsablauf optimiert und die Produktivität insgesamt erhöht werden, sodass steigende Bedarfsmengen leichter abgedeckt werden können. Darüber hinaus könnte der Einsatz eines flexiblen Serviceroboters dazu beitragen, Monotonie abzubauen (Schenk und Elkmann 2012). Unter der Voraussetzung eines flexiblen Robotersystems und der Prämisse eines gewissen Aufwands zum Erlernen der neuen technischen Möglichkeiten ist z. B. denkbar, dass sich der Mitarbeiter manuell auszuführende Arbeitstätigkeiten aktiv aussucht und die übrigen Schritte an das Robotersystem delegiert. Durch diese Form der Kooperation mit dem industriellen Serviceroboter kann der Anteil monotoner Aufgaben reduziert und gleichzeitig eine flexiblere Arbeitsstrukturierung erreicht werden (Hirsch-Kreinsen 2014).

Die betriebliche Rolle des Instandhalters wird durch einen steigenden Umsetzungsgrad industrieller Servicerobotik im Unternehmen stark beeinflusst. Zunächst ergeben sich vor diesem Hintergrund Änderungen, indem der Leistungsumfang der Instandhaltung um die in der Kleinteilemontage implementierten, neuen Robotersysteme erweitert wird. Hierdurch steigt die Komplexität der Arbeitsaufgabe und der Mitarbeiter wird zunehmend mit neuartigen Problemen konfrontiert. Dies betrifft u. a. die geforderten Kenntnisse und Fähigkeiten im Bereich der Informationstechnologien, wodurch ggf. eine bedarfsgerechte Befähigung und Qualifizierung der Mitarbeiter erforderlich wird (Molzow-Voit et al. 2015). Das umfasst 


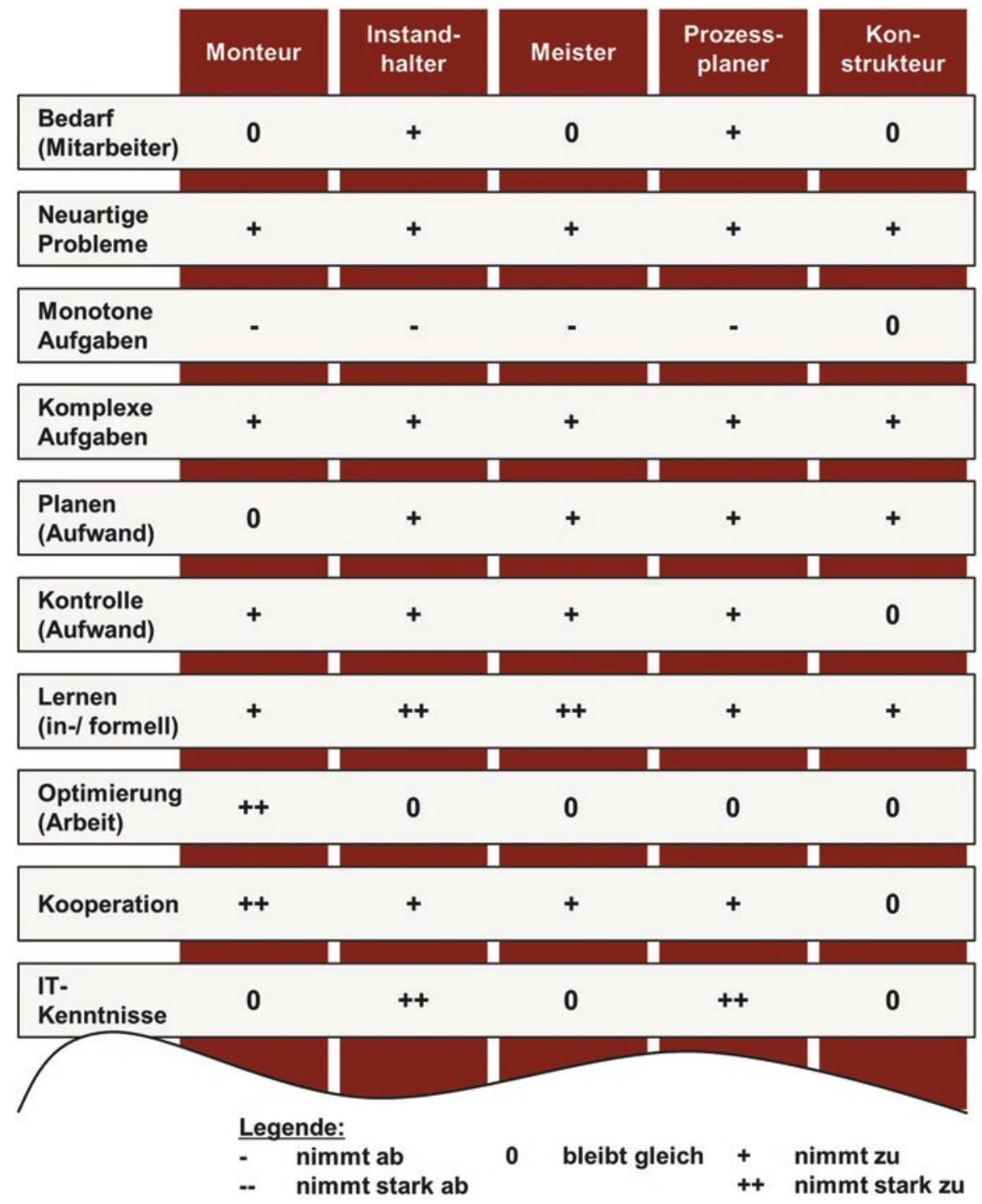

Abb. 4.4 Mögliche Auswirkungen auf betriebliche Rollen

sowohl die klassische (formelle) Aus- und Weiterbildung von aktuellen und zukünftigen Mitarbeitern als auch das Lernen am konkreten Prozess (informell). Ferner erhöht sich die Gesamtzahl technischer Systeme, welche potenziell der Instandhaltung unterliegen, was zu einer Erhöhung des Mitarbeiterbedarfs in diesem Arbeitsfeld führen kann.

Für die Rolle des Meisters ergeben sich umfassende Auswirkungen, die zu einem großen Teil auf die interaktive Rolle des Roboters in der Montage zurückzuführen sind. Im ersten Schritt entsteht zusätzlicher Schulungsbedarf für die entsprechenden 
Mitarbeiter, um die Grundlagen industrieller Servicerobotik sowie die daraus erwachsenden innovativen Chancen und Potenziale zu verstehen. Daran anknüpfend kann der Meister seine Mitarbeiter aus dem Bereich der Montage direkt in den Veränderungsprozess einbinden, indem er das gewonnene Wissen mit ihnen teilt und so möglichen Ängsten und hieraus entstehenden neuartigen Problemen vorbeugt (Sanders und Wulfsberg 2015).

Unter der Rolle des Prozessplaners werden in diesem Kontext indirekte Aufgaben verstanden, die zur Neuplanung oder Anpassung eines Arbeitsablaufs für einen spezifischen Arbeitsplatz, wie z. B. dem adressierten Anwendungsfall, erforderlich sind. Als fundamentale Voraussetzung zur Planung hybrider Arbeitsprozesse entsteht für Mitarbeiter in diesem Bereich ebenfalls ein spezifischer Qualifizierungsbedarf, speziell in Bezug auf neue softwaretechnische Planungswerkzeuge. Darüber hinaus ist die entstehende Planungsaufgabe im Vergleich zu rein manuellen Prozessen ungleich komplexer, was in Summe zu einem erhöhten Bedarf gut ausgebildeter, qualifizierter Mitarbeiter führen kann (Hirsch-Kreinsen 2014). Unabhängig davon sind diese Experten allerdings imstande, die spezifischen Fähigkeiten und Potenziale industrieller Serviceroboter zu berücksichtigen und so die individuellen Arbeitsausführungen von Mensch und Maschine zur Ermöglichung einer Kooperation effizient miteinander zu verknüpfen.

Für Mitarbeiter aus dem Bereich der Konstruktion werden sich in diesem Kontext ähnliche Anforderungen ergeben. Zunächst entsteht auch hier ein gewisser formeller Qualifizierungsbedarf, um ein grundlegendes Verständnis servicerobotischer Systeme durch die Mitarbeiter abzusichern. Außerdem steigt die Komplexität der Konstruktionsaufgabe insgesamt, da im Zuge der Bauteilgestaltung und Konstruktion ebenfalls automatisierungstechnische Anforderungen zu berücksichtigen sind.

\subsection{Fazit}

Anhand des skizzierten Anwendungsszenarios wurden zahlreiche mögliche Auswirkungen auf verschiedene betriebliche Rollen identifiziert und bewertet. Die vorgestellten Änderungen lassen sich zum großen Teil auf die neue Funktion des kooperierenden Robotersystems in der Produktion und nicht ausschließlich auf die spezifische Ausprägung des Anwendungsfalls zurückführen. Daher besteht die grundsätzliche Möglichkeit, dass die spezifizierten Auswirkungen zu einem gewissen Anteil in dieser oder ähnlicher Form auch auf andere industrielle Bereiche und Einsatzfelder übertragbar sind. In diesem Kontext sind in Abb. 4.5 die wesentlichen Auswirkungen zusammenfassend aufgeführt und anhand des REFA-Arbeitssystems strukturiert (REFA Bundesverband e.V. 2013). In Summe wird hierdurch nochmals der zentrale und weiter zunehmende Stellenwert der industriellen Servicerobotik für zahlreiche innerbetriebliche Anwendungsfelder herausgestellt. 


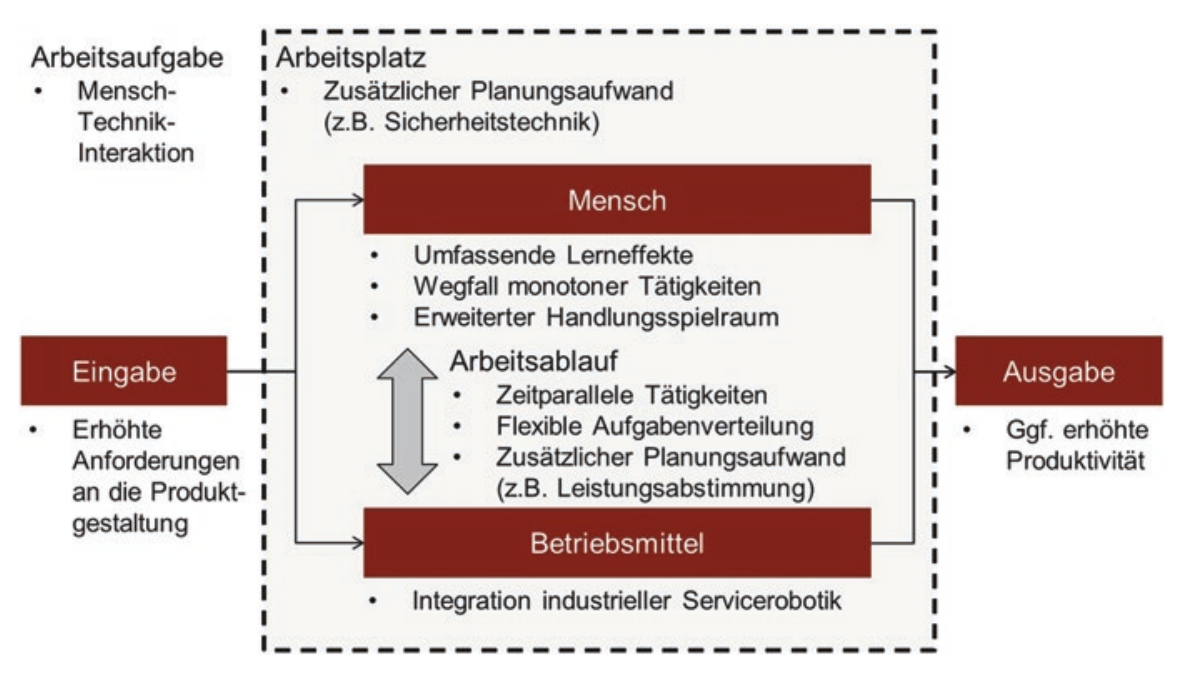

Abb. 4.5 Zusammenfassende Betrachtung der Auswirkungen

Um die fortschreitende Etablierung (teil-)automatisierter Systeme und die zunehmende Integration industrieller Servicerobotik in realen Anwendungsszenarien weiter zu unterstützen, kann eine Vielzahl unterschiedlicher Forschungs- und Entwicklungsansätze verfolgt werden. Ein zentraler Ansatzpunkt ist dabei die Entwicklung individueller Assistenzsysteme, welche an die jeweiligen Bedürfnisse und die individuelle Leistungsdisposition einzelner Mitarbeiter angepasst werden können. Der demografische Wandel und die damit einhergehende Änderung des Erwerbspersonenpotenzials bedingen eine stark schwankende inter- und intrapersonelle Leistungsfähigkeit. Jene möglichen individuellen alters-, verletzungs- und krankheitsbedingten Einschränkungen werden derzeit bei der Gestaltung hybrider Arbeitssysteme nur unzureichend betrachtet. Es gilt daher zukünftig, die individuelle Leistungsdisposition des Mitarbeiters detailliert zu erfassen und für den Roboter lesbar zu machen. In Abhängigkeit des jeweiligen Unterstützungsbedarfs und unter Zuhilfenahme neuer Planungswerkzeuge (z. B. individuelle digitale Menschmodelle) können Lösungen der direkten Mensch-Roboter-Kollaboration implementiert werden, um sowohl physische Leistungseinschränkungen zu kompensieren als auch um eine möglichst hohe Produktivität zu gewährleisten.

\subsection{Danksagung}

Das diesem Bericht zugrunde liegende Forschungs- und Entwicklungsprojekt wird mit Mitteln des Bundesministeriums für Wirtschaft und Energie (BMWi) innerhalb des Technologieprogramms „AUTONOMIK für Industrie 4.0“ unter dem Förderkennzeichen 01MA13011A gefördert und vom Projektträger „Technische Innovationen in der 
Wirtschaft" im Deutschen Zentrum für Luft- und Raumfahrt, Köln betreut. Die Verantwortung für den Inhalt dieser Veröffentlichung liegt beim Autor. Darüber hinaus möchten wir den beteiligten Projektpartnern Albrecht Jung GmbH \& Co. KG (Lünen), GEA Farm Technologies GmbH (Bönen), ICARUS Consulting GmbH (Lüneburg) und KHS Corpoplast GmbH (Hamburg) danken.

\section{Literatur}

Beumelburg, K. (2010). Fähigkeitsorientierte Montageablaufplanung in der direkten Mensch-Roboter-Kooperation. Heimsheim: Jost-Jetter.

BGIA. (2011). BG/BGIA-Empfehlungen für die Gefährdungsbeurteilung nach Maschinenrichtlinie - Gestaltung von Arbeitsplätzen mit kollaborierenden Robotern. Sankt Augustin: BGIA.

Bundesministerium für Wirtschaft und Technologie. (2013). Band 4 - Industrielle Servicerobotik Studie. Berlin: LoeschHundLiepold.

Deuse, J., Roßmann, J., Kuhlenkötter, B., Hengstebeck, A., Stern, O., \& Klöckner, M. (2014a). A methodology for the planning and implementation of service robotics in industrial work processes. In M. Putz, R. Mauermann, M. Pfeifer, \& P. Blau (Hrsg.), 5th CATS2014 - CIRP Conference on Assembly Technologies and Systems (S. 41-46). Elsevier, Amsterdam.

Deuse, J., Weisner, K., Hengstebeck, A., \& Busch, F. (2014b). Gestaltung von Produktionssystemen im Kontext von Industrie 4.0. In Bundeministerium für Wirtschaft und Technologie (Hrsg.), Zukunft der Arbeit in Industrie 4.0 (S. 43-49). Berlin: LoeschHundLiepold.

Deutsches Institut für Normung e.V. (2008). Ergonomie - Computer-Manikins und KörperumrissSchablonen. Deutsches Institut für Normung e.V. Berlin: Beuth.

Deutsches Institut für Normung e.V. (2010). 3D-Scanverfahren für international kompatible anthropometrische Datenbanken. Deutsches Institut für Normung e.V. Berlin: Beuth.

Deutsches Institut für Normung e.V. (2012). Industrieroboter - Sicherheitsanforderungen. Deutsches Institut für Normung e.V. Berlin: Beuth.

Hirsch-Kreinsen, H. (2014). Welche Auswirkungen hat "Industrie 4.0" auf die Arbeitswelt? Bonn: Friedrich-Ebert-Stiftung.

International Organization for Standardization. (2016). Robots and robotic devices - Collaborative industrial robots. International Organization for Standardization, Genf.

Klöckner, M., Weisner, K., Kuhlenkötter, B., Deuse, J., \& Roßmann, J. (2015). Entwicklung eines Werkzeuges zur anwendungsfallorientierten Auswahl servicerobotischer Systeme. In T. Bertram (Hrsg.), Fachtagung Mechatronik 2015 (S. 1-6). VDI Verlag, Düsseldorf.

Molzow-Voit, F., Quandt, M., Freitag, M., \& Spöttl, G. (2015). Qualifizierung von Fachkräften und Entscheidern - Einsatz von Robotikanwendungen in logistischen Arbeitsprozessen. Zeitschrift für wirtschaftlichen Fabrikbetrieb, 110, 583-586.

Ohno, T. (2009). Das Toyota-Produktionssystem. Frankfurt/New York: Campus Verlag.

Parasuraman, R., Sheridan, T. B., \& Wickens, C. D. (2000). A model for types and levels of human interaction with automation. IEEE Transactions on Systems, Man and Cybernetics, 30, 286-297.

REFA Bundesverband e.V. (2013). REFA. Kompakt-Grundausbildung 2.0 - Band 1. Weinheim: Druckhaus Diesbach.

Ross, P. (2002). Bestimmung des wirtschaftlichen Automatisierungsgrades von Montageprozessen in der frühen Phase der Montageplanung. München: H.-Utz-Verlag.

Sanders, A., \& Wulfsberg, J. (2015). Industrie 4.0: Shopfloor Management im Wandel - Konzeptionelle Handlungsempfehlungen. Zeitschrift für wirtschaftlichen Fabrikbetrieb, 110, 653-656. 
Schenk, M., \& Elkmann, N. (2012). Sichere Mensch-Roboter-Interaktion: Anforderungen, Voraussetzungen, Szenarien und Lösungsansätze. In E. Müller (Hrsg.), Demographischer Wandel - Herausforderungen für die Arbeits- und Betriebsorganisation der Zukunft (S. 109-122). GITO, Berlin.

Schlick, C., Bruder, R., \& Luczak, H. (2010). Arbeitswissenschaft. Berlin/Heidelberg: Springer.

Schraft, R. D., Degenhart, E., \& Hagele, M. (1993). Service robots: the appropriate level of automation and the role of users/operators in the task execution. In IEEE (Hrsg.), Systems Man and Cybernetics (S. 163-169). IEEE, New York.

Spingler, J., \& Thiemermann, S. (2001). Direkte Mensch-Roboter-Kooperation - Am Beispiel einer flexiblen Montagezelle. Zeitschrift für wirtschaftlichen Fabrikbetrieb, 96, 616-619.

Steegmüller, D., \& Zürn, M. (2014). Industrie 4.0 in Produktion, Automatisierung und Logistik. Wiesbaden: Springer.

Universal Robots. (2014). Schnell zum Start bereit - Leichtbauroboter nun überarbeitet. Handling Handhabungstechnik - Fabrikautomation; Intralogistik, 9, 88-89.

Open Access Dieses Kapitel wird unter der Creative Commons Namensnennung 4.0 International Lizenz (http://creativecommons.org/licenses/by/4.0/deed.de) veröffentlicht, welche die Nutzung, Vervielfältigung, Bearbeitung, Verbreitung und Wiedergabe in jeglichem Medium und Format erlaubt, sofern Sie den/die ursprünglichen Autor(en) und die Quelle ordnungsge-mäß nennen, einen Link zur Creative Commons Lizenz beifügen und angeben, ob Änderungen vorgenommen wurden.

Die in diesem Kapitel enthaltenen Bilder und sonstiges Drittmaterial unterliegen ebenfalls der genannten Creative Commons Lizenz, sofern sich aus der Abbildungslegende nichts anderes ergibt. Sofern das betreffende Material nicht unter der genannten Creative Commons Lizenz steht und die betreffende Handlung nicht nach gesetzlichen Vorschriften erlaubt ist, ist für die oben aufgeführten Weiterverwendungen des Materials die Einwilligung des jeweiligen Recht-einhabers einzuholen.

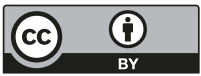

